\title{
First-Principle Study of Electronic Structure and Magnetic Properties of $\mathrm{Tb}_{2} \mathrm{FeCrO}_{6}$
}

Sarita Lawaju $\mathbf{u}^{1,2}$, Shalika Ram Bhandari ${ }^{1,2}$, Santosh K. C. ${ }^{3}$, and Madhav Prasad Ghimire ${ }^{1,2^{*}}$

${ }^{1}$ Central Department of Physics, Tribhuvan University, Kirtipur, 44613, Kathmandu, Nepal

${ }^{2}$ Condensed Matter Physics Research Center, Butwal-11, Rupandehi, Nepal ${ }^{3}$ San José State University, San Jose, California, United States

* madhav.ghimire@cdp.tu.edu.np 


\section{Outline}

- Abstract

- Introduction and Motivation

- Crystal Structure

- Computational Details

- Results and Discussions

- Conclusions 


\section{Abstract}

Electronic and magnetic properties of $\mathrm{Tb}_{2} \mathrm{FeCrO}_{6}$ (TFCO) is investigated using density functional theory (DFT). TFCO shows the insulating property with the band gap of $0.048 \mathrm{eV}$ and 2.372 $\mathrm{eV}$ with generalized gradient approximation (GGA) and GGA + U respectively. The antiferromagnetic configuration, AFM1 $\left[\mathrm{Fe}_{1} \mathrm{Fe}_{2} \mathrm{Cr}_{1} \mathrm{Cr}_{2}-\uparrow \downarrow \uparrow \downarrow\right]$ is found to be the ground state. Further, exchange coupling constants $\mathrm{J}_{\mathrm{Cr}-\mathrm{Cr}}(-0.99 \mathrm{meV}), \mathrm{J}_{\mathrm{Fe}-\mathrm{Fe}}(-5.68 \mathrm{meV})$, and $\mathrm{J}_{\mathrm{Cr}-\mathrm{Fe}}(-1.36 \mathrm{meV})$ are calculated to get depth knowledge on magnetic realm of TFCO.

Keywords: Density functional theory; generalized gradient approximation; antiferromagnetic; magnetic exchange interaction 


\section{Introduction and Motivation}

- Double perovskite (DP) is the compound having general formula $\mathrm{A}_{2} \mathrm{BB}^{\prime} \mathrm{O}_{6}$, where $\mathrm{A}$ - rare earth element; $\mathrm{B}, \mathrm{B}^{\prime}$ transition elements.

- DPs containing $\mathrm{Fe}$ and $\mathrm{Cr}$ shows interesting properties like multiferroicity and can have varieties of applications. ${ }^{1}$

- Experimentally, TFCO showed re-entrant spin reorientation and griffith's phase transition. ${ }^{2}$

- TFCO-Pbnm structure has G-type antiferromagnetic ground state and shows insulating property. ${ }^{2}$

- Spin-Heisenberg Hamiltonian: $\hat{\mathbf{H}}_{\text {spin }}=-\sum_{\mathbf{i}<\mathrm{j}} \mathbf{J}_{\mathrm{ij}} \hat{\mathbf{S}}_{\mathrm{i}} \hat{\mathbf{S}}_{\mathbf{j}}$ 


\section{Crystal Structure}

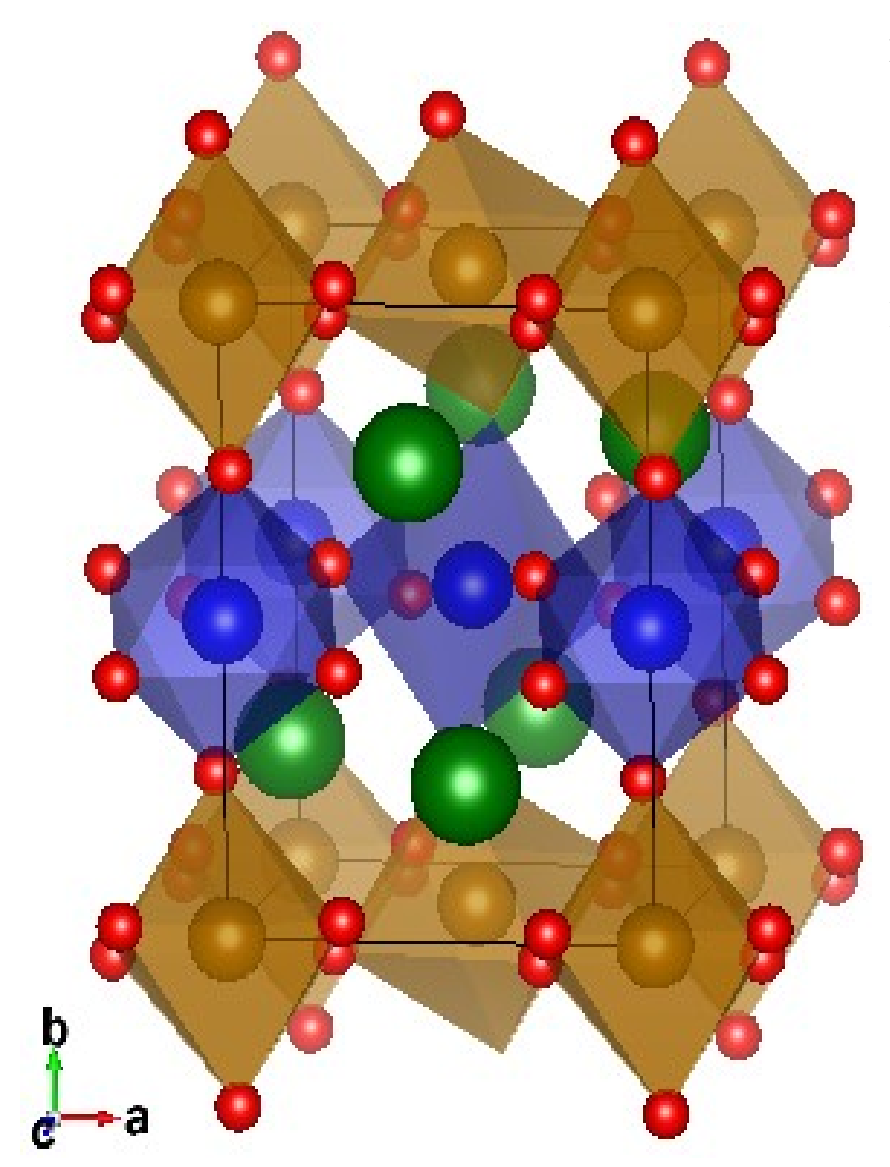

> $\mathrm{Tb}_{2} \mathrm{FeCrO}_{6}$ - space group (62 - Pnma), orthorhombic

- Lattice parameters:

$$
\begin{aligned}
& a=5.53913 \AA, b=7.59173 \AA, \\
& c=5.31242 \AA \text { and } \\
& \alpha=\beta=y=90^{\circ}
\end{aligned}
$$

- Charge state: $\mathrm{Tb}\left(4 \mathrm{f}^{8}\right) \longrightarrow+3$,

$$
\begin{aligned}
& \mathrm{Fe}\left(3 d^{5}\right) \longrightarrow+3 \\
& \mathrm{Cr}\left(3 d^{3}\right) \longrightarrow+3
\end{aligned}
$$

- Neel's temperature (experimental):

$$
\text { - } \mathrm{T}_{\mathrm{N}}=257 \mathrm{~K}
$$

Figure: Crystal structure of

$\mathrm{Tb}_{2} \mathrm{FeCrO}_{6}$ (Pnma)

$$
>\mathrm{S}_{\mathrm{Fe}}=\frac{5}{2}, \quad \mathrm{~S}_{\mathrm{Cr}}=\frac{3}{2}
$$




\section{Computational Details}

- Used density functional theory (DFT) using FP-LAPW + lo method implemented in WIEN2k code. ${ }^{3}$

> $\mathrm{R}_{\mathrm{MT}}$ values for $\mathrm{Tb}, \mathrm{Fe}, \mathrm{Cr}$ and $\mathrm{O}$ are 2.35, 2.0, 1.96 and 1.72 Bohr respectively.

- Implemented generalized gradient approximation (GGA) and hubbard potential $(+U)$.

> On-site potential values used are: $\mathrm{U}_{\mathrm{Tb}}=6 \mathrm{eV}, \mathrm{U}_{\mathrm{Fe}}=5 \mathrm{eV}$, and $\mathrm{U}_{\mathrm{Cr}}=3 \mathrm{eV}$.

- Self-convergence criteria: energy convergence - 10-5 Ry, charge convergence $-10^{-4} \mathrm{e}$.

> $6 \times 6 \times 6$ k - mesh over irreducible Brillouin Zone (BZ). 


\section{Results and Discussions}

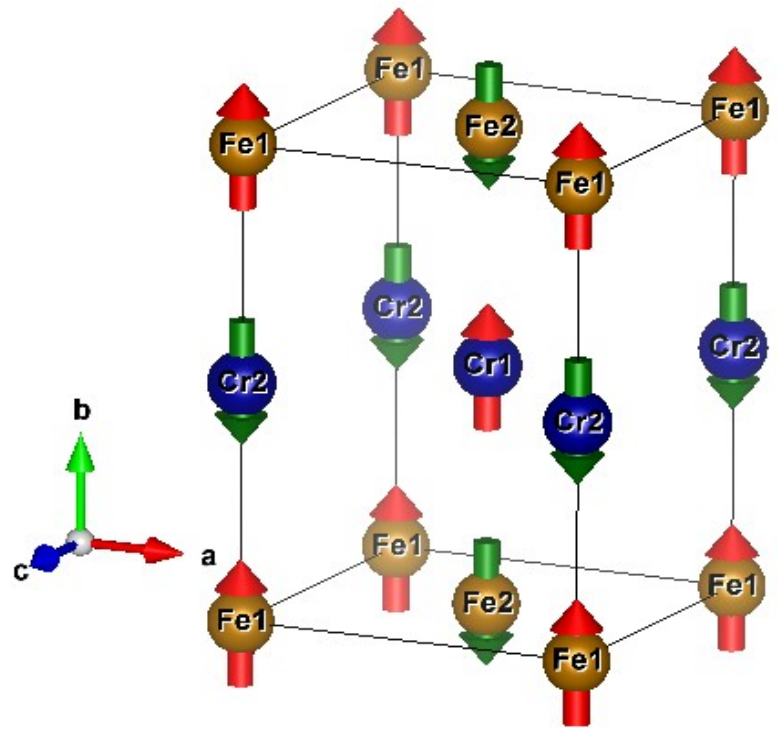

Figure: Ground state spin configuration of TFCO-Pnma
- Optimized position of 3d-transition elements is in order $\mathrm{Fe}_{1} \mathrm{Fe}_{2} \mathrm{Cr}_{1} \mathrm{Cr}_{2}$.

- Up/down spins of magnetic ions $\mathrm{Fe}_{1}, \mathrm{Fe}_{2}, \mathrm{Cr}_{1}, \mathrm{Cr}_{2}$ ordering are: FM- $\uparrow \uparrow \uparrow \uparrow, A F M 1 . \uparrow \uparrow \downarrow$, AFM2- $\uparrow \downarrow \downarrow \uparrow$, FIM1- $\uparrow \downarrow \downarrow, ~ F I M 2-\uparrow \downarrow \downarrow \downarrow$

- Spin ground state configuration AFM1- $\uparrow \downarrow \uparrow \downarrow$ (G-Type AFM).

> Magnetic moments: Fe(GGA) $= \pm 3.67 \mu_{\mathrm{B}}, \mathrm{Cr}(\mathrm{GGA})= \pm 2.36 \mu_{\mathrm{B}}$

$$
\mathrm{Fe}(\mathrm{GGA}+\mathrm{U})= \pm 4.12 \mu_{\mathrm{B}}, \quad \mathrm{Cr}(\mathrm{GGA}+\mathrm{U})= \pm 2.57 \mu_{\mathrm{B}}
$$

> Present of band gap of 0.048 (2.372) eV within GGA (GGA + U). 


\section{Results and Discussions}

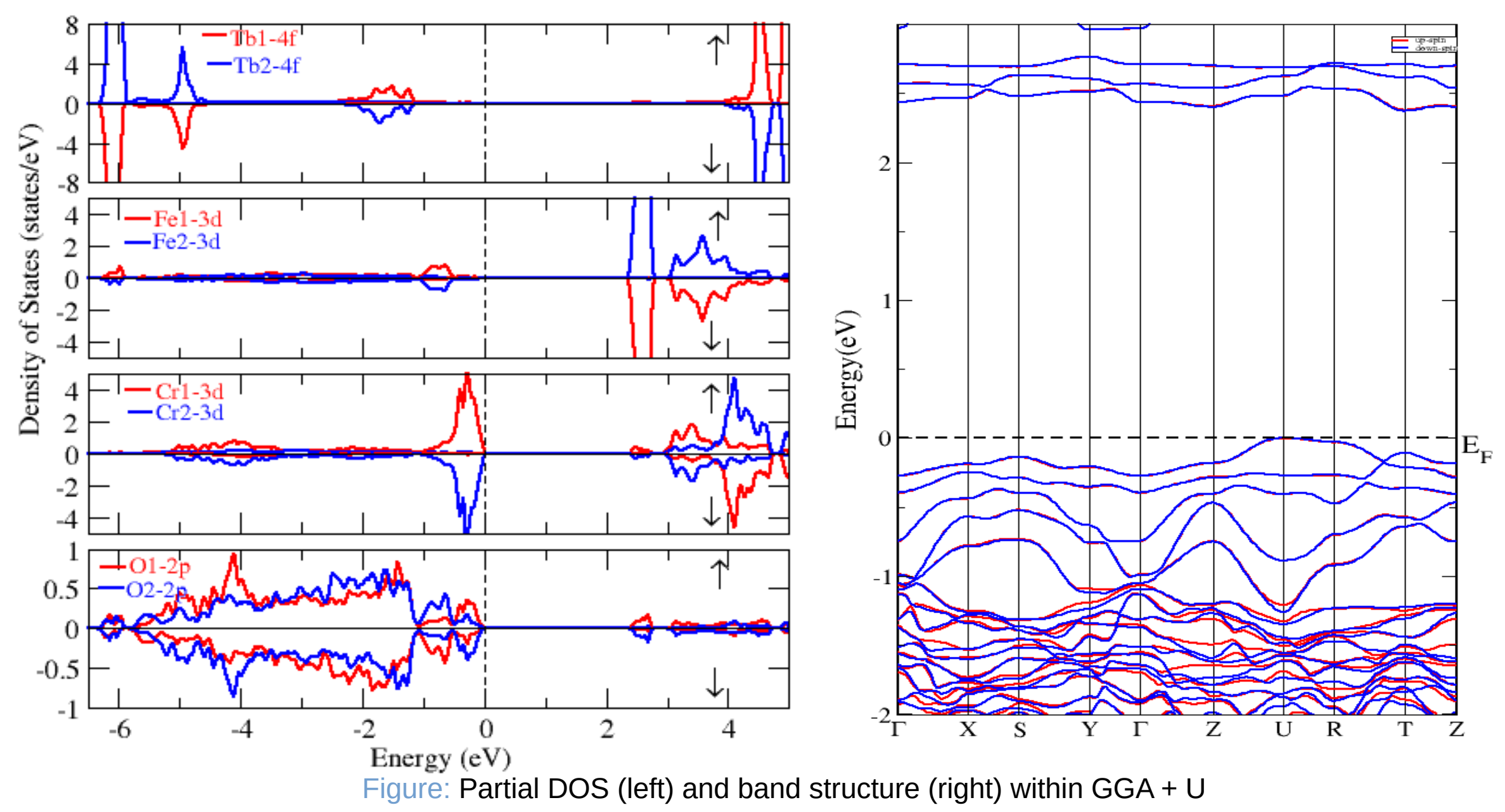

- Band gap is increased from $0.048 \mathrm{eV}$ (GGA) to $2.372 \mathrm{eV}$ (GGA+U).

- Gap is contributed by Cr-3d (valence region) and Fe-3d (conduction region). 


\section{Results and Discussions}

\section{Exchange coupling constants}

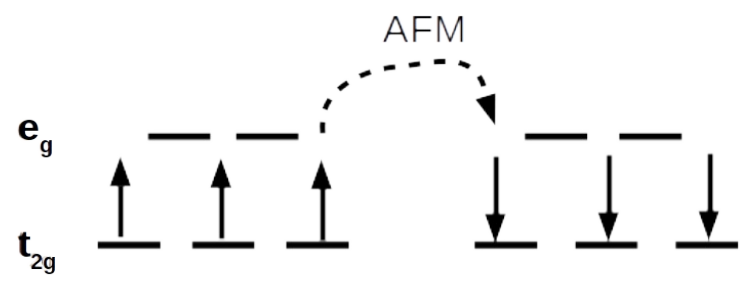

$\mathrm{Cr}_{1}$

$$
\mathrm{Cr}_{2}
$$

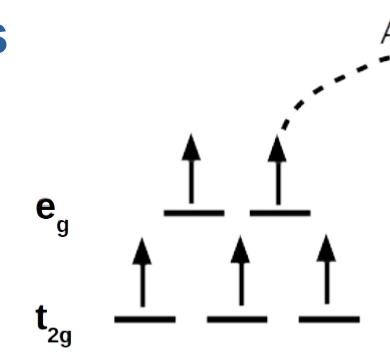

$\mathrm{Fe}_{1}$

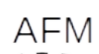

AFM

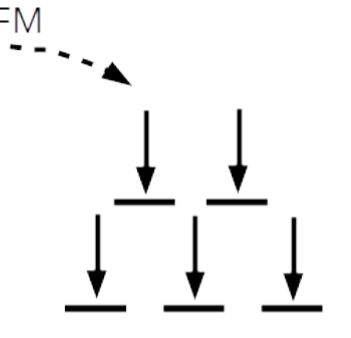

$\mathrm{Fe}_{2}$

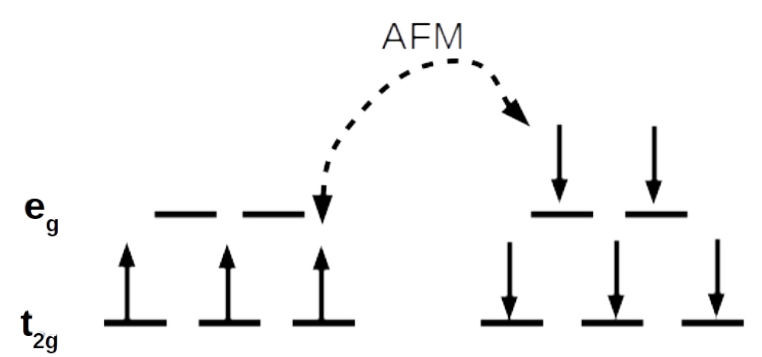

$\mathrm{Cr}$
$\mathrm{Fe}$

Figure: Super-exchange interactions between $\mathrm{Cr}$ atoms (left), $\mathrm{Fe}$ atoms (middle) and $\mathrm{Cr}$-Fe atoms (right)

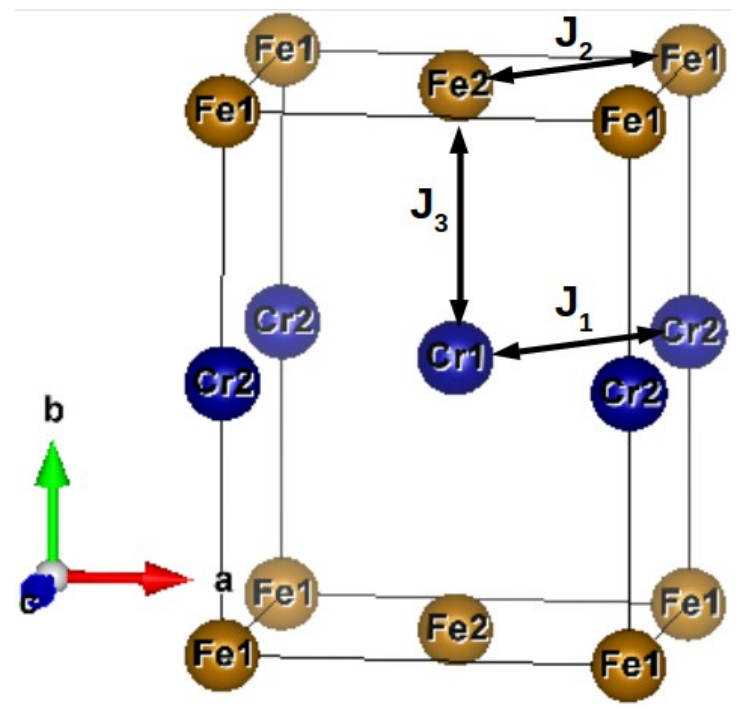

> $\mathrm{J}_{\mathrm{Cr}-\mathrm{Cr}}=-0.99 \mathrm{meV}$ : Anti-ferromagnetic order.

> $\mathrm{J}_{\mathrm{Fe}-\mathrm{Fe}}=-5.68 \mathrm{meV}$ : Anti-ferromagnetic order.

> $\mathrm{J}_{\mathrm{Cr}-\mathrm{Fe}}=-1.36 \mathrm{meV}$ : Anti-ferromagnetic order.

> The interaction between Fe atoms is stronger of all.

> Neel's temperature was theoretically calculated using formula:

$$
\mathrm{T}_{\mathrm{N}}=\frac{2 \mathrm{~S}_{\mathrm{Fe}} \mathrm{S}_{\mathrm{Cr}}}{3 \mathrm{~K}_{\mathrm{B}}}\left(4 \mathrm{~J}_{1}+4 \mathrm{~J}_{2}+2 \mathrm{~J}_{3}\right)
$$

Figure: $\mathrm{J}$ - path chosen for TBCO

$>\mathrm{T}_{\mathrm{N}}($ calculated $)=853.69 \mathrm{~K}$ 


\section{Conclusions}

> G - Type AFM1- $\uparrow \uparrow \downarrow\left[\mathrm{Fe}_{1} \mathrm{Fe}_{2} \mathrm{Cr}_{1} \mathrm{Cr}_{2}\right]$ is the spin ground state.

> Compound is an insulator with a band gap of 0.048 (2.372) eV within GGA (GGA+U).

> Gap is contributed by Cr-3d and Fe-3d states

- The exchange interactions between all transition elements are in antiferromagnetic order.

> Insulating behavior of compound shows possibilities of Multiferroicity in it and hence potential uses in spintronic devices, photo-voltaic devices, memory states, etc. 


\section{Acknowledgments}

University Grant Commission Nepal

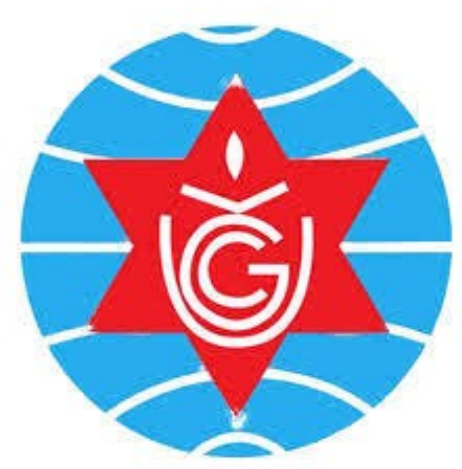

Condensed Matter Physics Research Center Butwal, Nepal

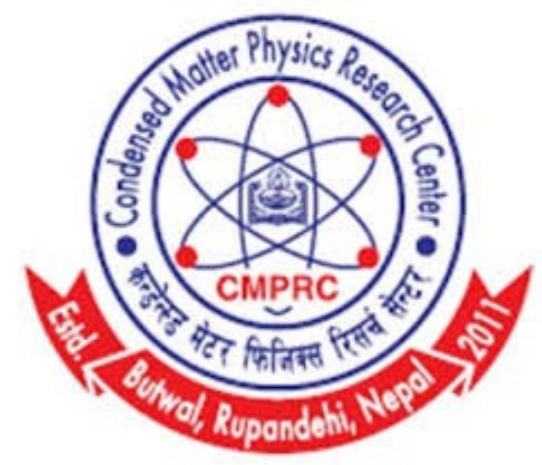

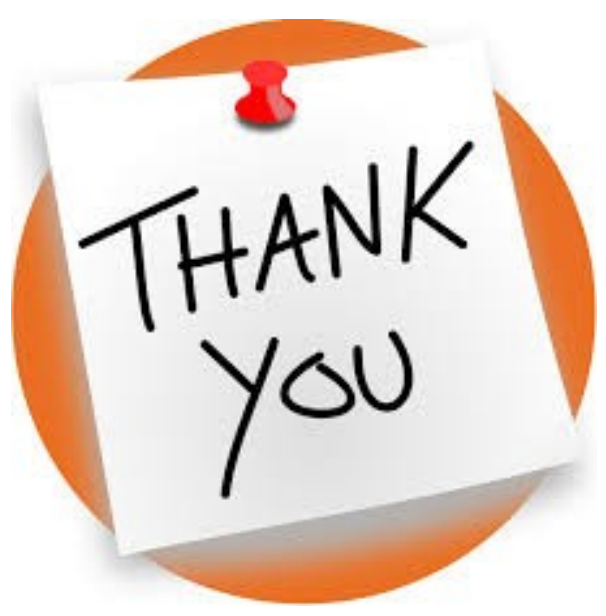

Crystals 2020 